\title{
Crystal structure and optical activity of $\mathrm{La}_{3} \mathrm{Ga}_{5} \mathrm{SiO}_{14}$ crystals
}

\author{
Shopa Ya., Ftomyn N. and Sokoliuk I. \\ Physics Faculty, Ivan Franko National University of Lviv, \\ 8 Kyrylo and Mefodiy Street, 79005 Lviv, Ukraine shopa@franko.lviv.ua
}

Received: 14.07.2014

\begin{abstract}
Using a laser polarimeter $(635 \mathrm{~nm})$, we have obtained a spatial distribution map of optical rotatory power (OR) on a $z$-cut sample of langasite crystals $\mathrm{La}_{3} \mathrm{Ga}_{5} \mathrm{SiO}_{14}$ with the transverse dimensions of $3 \times 17 \mathrm{~mm}^{2}$ and the thickness of $0.945 \mathrm{~mm}$. A possible impact of linear optical birefringence on the measured OR is clarified. Basing on polarizability theory of the optical activity effect, the OR is calculated as a function of disordering structure parameters of the material under investigation. It is shown that the structural disorder prevails in the spatial changes of OR along the optic axis.
\end{abstract}

Keywords: optical activity, polarizability, polarimetry, structural disorder

PACS: $61.50 . \mathrm{Ah}, 78.20 . \mathrm{Ek}, 78.20 . \mathrm{Fm}$

UDC: $535.51,535.56,538.911$

\section{Introduction}

In the recent years special attention has been paid to correlation between the structure and the optical properties of crystals such as refractive, gyrotropic, etc. Optical activity (OA) effect represents a phenomenon which is one of the most sensitive to the peculiarities of crystalline structure. The effect is described quantitatively by a so-called specific rotatory power $\rho=\pi\left(n_{L}-n_{R}\right) / \lambda$, which measures the specific rotation of light polarization plane (with $n_{L}$ and $n_{R}$ denoting the refractive indices for the left and right circularly polarized light and $\lambda$ the wavelength of probing light [1]). There is a number of precise experimental techniques used on order to measure the OR for different directions in crystals. Furthermore, there exist a possibility for calculating the OR with a polarizability theory of OA, issuing from the structural data for a given crystal and the information about electronic polarizabilities of its individual atoms [2].

$\mathrm{La}_{3} \mathrm{Ga}_{5} \mathrm{SiO}_{14}$ single crystals (abbreviated as LGS) belong to a large langasite family with a structural type of Ca-gallogermanate. This material, which is grown with a Czochralski technique [3], remains promising for quantum electronics, high-temperature sensors, etc. [4-6]. Langasite compounds have the space group symmetry $P 321$ (the point symmetry 32), with one molecule per unit cell. A very interesting structural feature of these crystals is their disordering: $2 d$ Wyckoff positions are partially occupied by Ga and Si atoms [7]. Moreover, the LGS crystals have a layered structure. There are different oxygen polyhedra: two types of tetrahedra (around the cations in the $2 d$ and $3 f$ positions; the symmetries 3 and 2), octahedra (the $1 a$ position; the symmetry 32 ), and distorted Thomson cubes (the $3 e$ position; the symmetry 2) $[4,7]$.

Optical properties of these materials have been extensively studied $[4,5,8,9]$. LGS is a noncentrosymmetric, optically uniaxial, and optically positive $\left(n_{e}>n_{o}\right)$ crystal. It is also optically active, with a diagonal gyration tensor $g_{i i}[4,5]$.

In this study we consider gyrotropic properties of LGS crystals. As mentioned before, they belong to disordered materials. According to earlier investigations of the crystal structure, the 
occupations of their $2 d$ positions are as follows: $0.5 \mathrm{Ga}+0.5 \mathrm{Si}$. It is well known that the optical parameters of these materials such as refractive indices or electrooptic coefficients depend strongly on their exact composition and spatial inhomogeneity (see, e.g., Refs. [10,11]). That is why we have decided to study possible correlations between the optical properties (both gyrotropic and refractive) and the peculiarities of structural disorder in these compounds.

As a result, the direct aim of the present work is to analyze dependence between the site occupancy of the $2 d$ Wyckoff positions for Ga and Si atoms and the optical activity of LGS. This has been accomplished via measuring the OR along the optic axis and applying a computation method known from Ref. [2].

\section{Experimental results}

First of all, we have constructed a map of spatial distribution for the OR parameter $\rho$ measured along the optic axis. A $z$-cut LGS sample was used, with the transverse dimensions $3 \times 17 \mathrm{~mm}^{2}$ and the thickness of $0.945 \mathrm{~mm}$. The experiments were performed with the aid of a computer-controlled laser polarimeter $(\lambda=635 \mathrm{~nm})$. A polarizer and an analyzer were Glan-type calcite prisms having the apertures of $10 \times 10 \mathrm{~mm}^{2}$. To change angular orientation of the analyzer, we used a rotation stage with a step motor. The angular resolution of this mechanism was $\sim 1.33 \cdot 10^{-3} \mathrm{deg}$ over the total angular range scanned as large as $10 \mathrm{deg}$.

The light intensity transmitted through the analyzer was detected using a photodiode FD-288A followed with a high-impedance operational amplifier AD8646 and a 24-bit ADC connected to a PC. In a photovoltaic mode, the photodiode generated a small current proportional to the intensity level $I$ over 6 to 9 decades [12]. Communication between the 24-bit ADC and the PC-control software was handled via an LPT port.

In our experiments we measured from 9 to 15 data points of the light intensity $I$ (ADC codes) as a function of the analyzer angle $\alpha$ in the range of $\sim 1^{\circ}$. When the angular orientation of analyzer deviates by a small angle $\alpha$ from that corresponding to the condition of perfectly crossed polarizers, the transmitted light intensity $I$ can be found using the Malus's law (see $[1,13]$ ):

$$
I(\alpha)=I_{0}\left(\sin ^{2} \alpha+\varepsilon^{2}\right),
$$

where $I_{0}$ implies the maximum transmitted intensity, $\varepsilon$ the light ellipticity that depends on the quality of polarization system employed. In our case, the latter mainly depends on small linear birefringence existing along the optic axis. For $\alpha \ll 1$ we have $\sin ^{2} \alpha \approx \alpha^{2}$, so that Eq. (1) may be written in a parabolic form:

$$
I(\alpha)=a \alpha^{2}+b \alpha+c .
$$

After least-square fitting of the experimental data $I(\alpha)$ one can obtain three parabolic parameters entering in Eq. (2) and then determine the parameters $\alpha_{\min }=-b / 2 a$ and $\varepsilon^{2}=c / a-b^{2} / 4 a^{2}$.

The main source of the linear birefringence available along the optic axis in crystals grown with the Czochralski method is so-called residual mechanical stresses. They remain in crystalline boules after they are cooled down. To determine the birefringence in a selected point of crystal sample, we measured 12 data points for the $\varepsilon$ parameter while rotating the polarizer by $360^{\circ}$ with the increments of $30^{\circ}$ (see Fig. 1). Consider a plate with a thickness $d$ cut from optically active crystal. Whenever a circular birefringence $\Delta n_{c}$ and a small linear birefringence $\Delta n_{l}$ coexist in this crystal, the ellipticity $\varepsilon$ of transmitted light is given by $[14,15]$ 


$$
\sin 2 \varepsilon=\cos 2 \gamma[(1-\cos \Gamma) \sin 2 \gamma \cos 2 \theta+\sin 2 \theta \sin \Gamma] .
$$

Here $\theta$ is the azimuth of linearly polarized incident light, $\Gamma=2 \pi \Delta n d / \lambda$ the phase difference, $\Delta n=\sqrt{\Delta n_{l}^{2}+\Delta n_{c}^{2}}$ the total (elliptical) birefringence, and $\gamma=0.5 \arctan \left(\Delta n_{c} / \Delta n_{l}\right)$. Since the $\varepsilon$ values in our experiments are small, further on we can assume that $\sin 2 \varepsilon \approx 2 \varepsilon$.

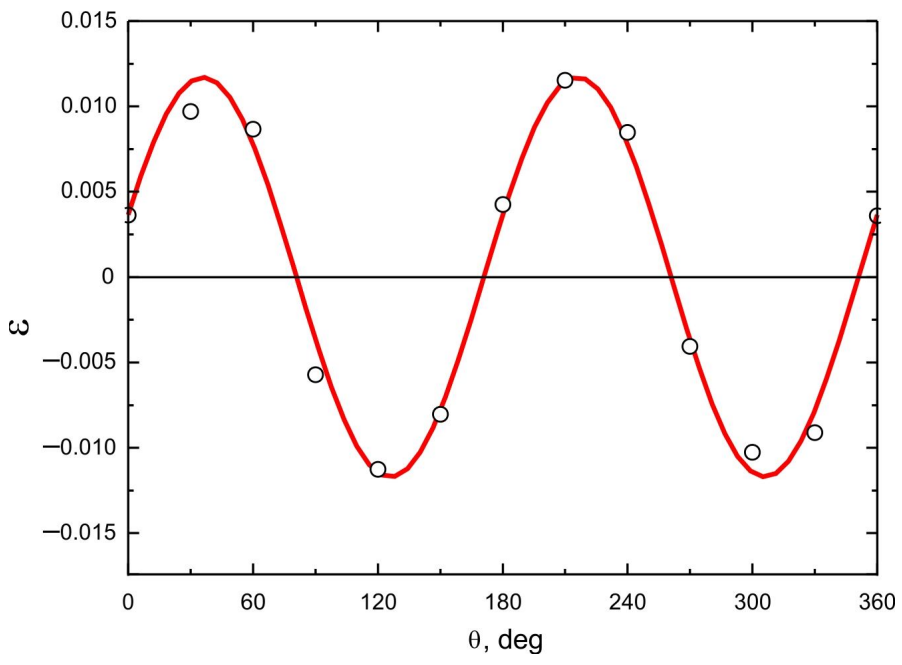

Fig. 1. Typical dependence of light ellipticity $\varepsilon$ on the polarizer angle $\theta$, with the $\varepsilon$ sign taken into account. Open circles correspond to experiment and solid curve to least-squares fit based on Eq. (3). The latter enables determining $\Gamma$ parameter and then linear birefringence $\Delta n_{l}$ induced along the optic axis by residual stresses.

Using the $\Delta n_{c}$ values known for LGS and the dependence $\varepsilon(\theta)$ measured experimentally (see Fig. 1), we have estimated the linear birefringence along the optic axis as $\Delta n_{l}=2.4 \cdot 10^{-6}$ and the phase difference as $\Gamma=0.117$. Basing on that data, one can estimate the influence of elliptical birefringence $\Delta n$ on the measured value of the OR $\rho$. Under the same conditions as in Eq. (3), the expression for the polarization azimuth $\chi$ has the following form $[14,15]$ :

$$
\tan 2 \chi=\frac{\sin 2 \theta \cos \Gamma+\sin 2 \gamma \cos 2 \theta \sin \Gamma}{\left(\cos ^{2} 2 \gamma+\sin ^{2} 2 \gamma \cos \Gamma\right) \cos 2 \theta-\sin 2 \gamma \sin 2 \theta \sin \Gamma} .
$$

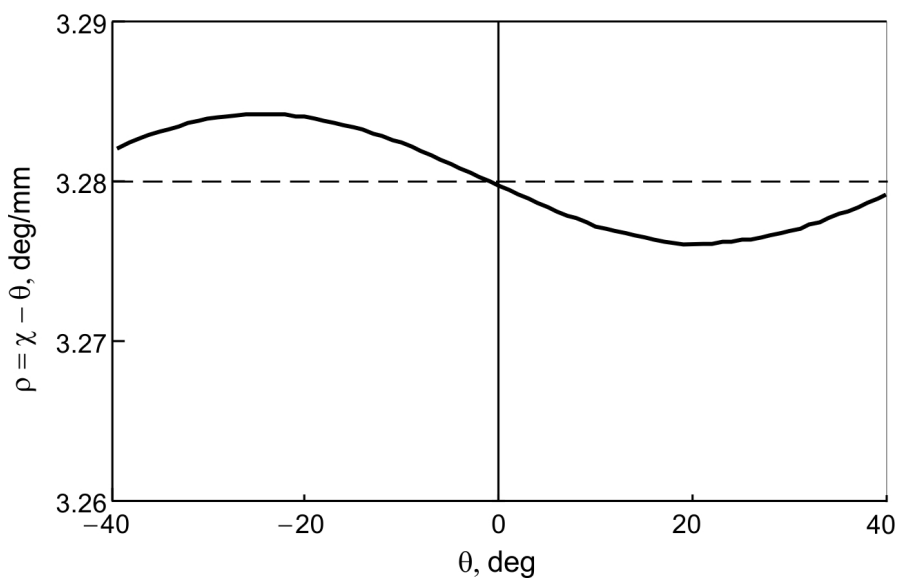

Fig. 2. Dependence of specific angular difference $\rho=x-\theta$ on the $\theta$ angle calculated using Eq. (4) for LGS plate with $d=1 \mathrm{~mm}, \lambda=635 \mathrm{~nm}$ and $\Gamma=0.117$. Azimuth $\theta$ is read from the polarizer's position corresponding approximately to the minimal $\varepsilon$. 
As seen from Fig. 2, the specific OR $\rho$ is only slightly dependent on the azimuth $\theta$ in the presence of small linear birefringence along the optic axis direction. The maximum deviation from its nominal value $\rho=3.28 \mathrm{deg} / \mathrm{mm}$ does not exceed $\Delta \rho \approx \pm 0.005 \mathrm{deg} / \mathrm{mm}$. Although we have made these calculations for a single selected point on the surface of sample, the $\Delta n_{l}$ value obtained by us and so the $\Gamma$ parameter can be regarded as being typical. Notice that they are determined by the parabolic coefficients of the dependence $I(\alpha)$, which actually define the linear birefringence.

As a second stage of our experimental studies, we have measured a grid of OR values with a $1 \mathrm{~mm}$ step along the $X$ and $Y$ axes, using the same laser polarimeter. The resulting $\rho(x, y)$ map constructed after proper averaging and smoothing is shown in Fig. 3a. Fig. 3 confirms readily that the maximal deviations of the $\rho$ parameter from its nominal value measured experimentally are indeed within the limit of approximately $\Delta \rho \approx \pm 0.05 \mathrm{deg} / \mathrm{mm}$. The $\Delta n_{l}$ value obtained for the selected point is quite large as for stresses-induced birefringence, though it gives an order of magnitude smaller contribution to the observed spatial fluctuations of the OR. It seems to be hardly reasonable that the magnitude $\Delta n_{l}$ could have as large order of magnitude as $10^{-5}$, as at the extreme points seen in Fig. 3 (the azimuth variation being $\theta \approx \pm 22.5^{\circ}$ ). In our opinion, these OR fluctuations may first of all be associated with the structural disorder of the material, which is different at different points. The latter is to be further verified using calculations that follow below.
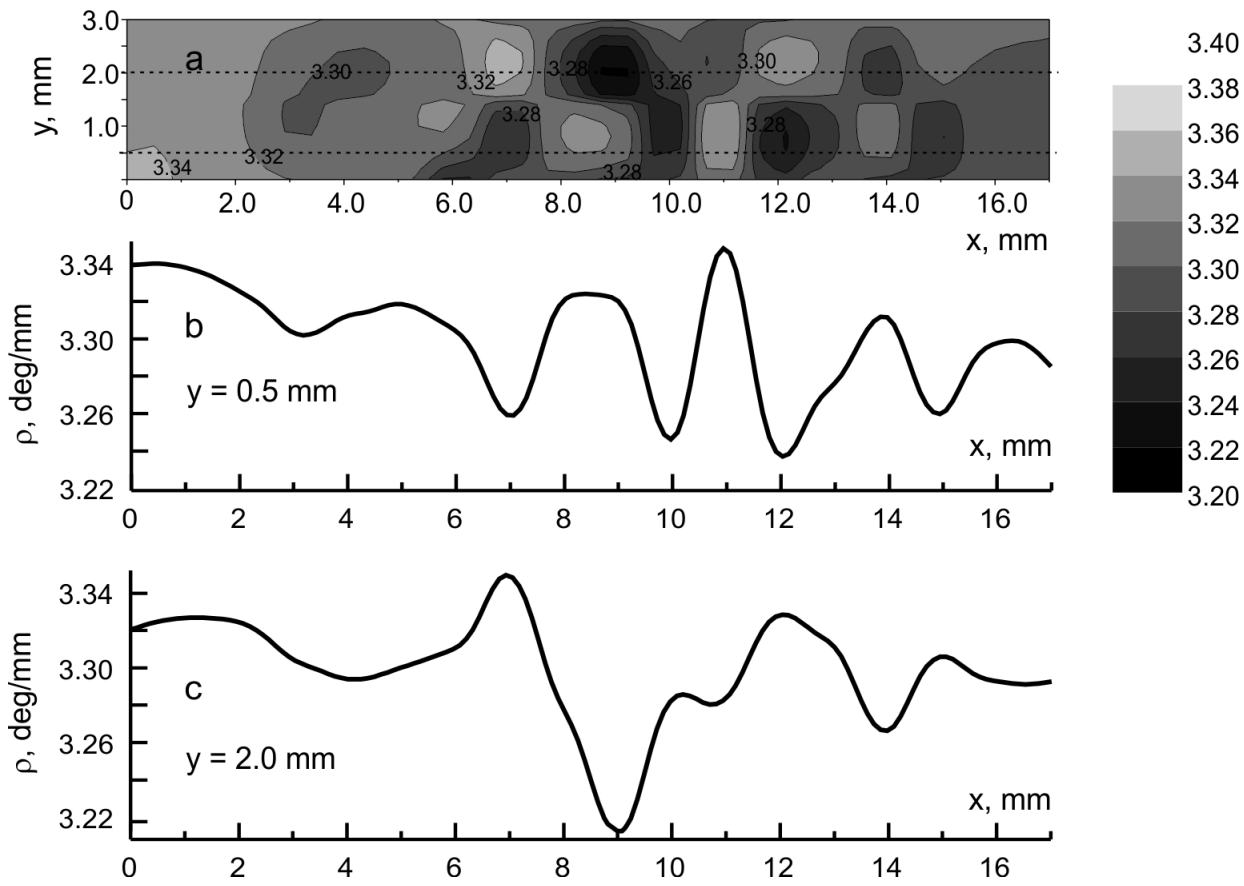

Fig. 3. Spatial distribution of OR $\rho$ measured along the optic axis in optically active LGS crystals at $\lambda=635 \mathrm{~nm}$ (panel a). Panels (b) and (c) show changes in the $\rho$ value as observed while scanning laser beam position along the $X$ axis: $y=0.5 \mathrm{~mm}$ (b) and $2.0 \mathrm{~mm}$ (c). The coordinate is read beginning from a lower edge of crystalline sample (see dashed lines in panel a).

\section{Calculation technique}

Below we consider the problem of calculating the OA [2] basing upon the dipole-dipole interaction theory [16]. First, we notice that the calculation method described in Ref. [2] could be applied to inorganic molecular or ionic crystals. Moreover, it is valid only for the visible spectral 
region where there are no absorption edges [2]. In general, the calculations need correct and very precise data on the crystalline structure (including the coordinates $\mathbf{r}_{S}$ of each of Sth atoms in the crystalline lattice) and the electronic polarizabilities $\alpha_{S}$ of the individual atoms (including the electronic polarizability volume $\alpha^{\prime}=\alpha / 4 \pi \varepsilon_{0}$, with $\varepsilon_{0}$ being the free-space permittivity).

According to the technique described in Ref. [2], the OR $\rho(\mathbf{k})$ as a function of wave vector $\mathbf{k}$ and the optical dielectric constants $\varepsilon_{i j}$ may be calculated using the formulae

$$
\begin{aligned}
& \varepsilon_{i j}=\delta_{i j}+\frac{1}{v} \sum_{S S^{\prime}}\left(C_{S S^{\prime}}\right)_{i j}, \\
& \rho(\mathbf{k})=-\frac{e_{r i j} k_{r}}{2 n v} \operatorname{Im} \sum_{S S^{\prime}}\left(C_{S S^{\prime}}\right)_{i j} .
\end{aligned}
$$

Here $S$ denotes the index of different atoms in the crystalline structure, $e_{r i j}$ the Levi-Civita symbol, $n$ the refractive index, $v$ the unit-cell volume, $\delta_{i j}$ the Kronecker symbol, and $C_{S S^{\prime}}$ imply some complex tensors. The $C_{S S^{\prime}}$ tensors are in general defined as functions of the electronic polarizability volume. The most convenient way to calculate these tensors is to use specific equations of the model (see Ref. [2]) and the information about the precise crystalline structure (as for the langasite crystals see Ref. [7]).

\section{Results of calculations}

We have performed the calculations for $635 \mathrm{~nm}$, which is just the wavelength of laser used in our polarimeter. The electronic polarizability volumes $\alpha^{\prime}$ for $\mathrm{La}^{3+}, \mathrm{Ga}^{3+}, \mathrm{Si}^{4+}$ and $\mathrm{O}^{2-}$ ions in the crystals under test have earlier been calculated in Ref. [17]. Those calculations have revealed that the electronic polarizability volumes of $\mathrm{Ga}^{3+}$ and $\mathrm{Si}^{4+}$ ions occupying the mixed atom sites $2 d$ vary in a limited range with respect to the occupancy factors reported, e.g., in the most recent structural data of Ref. [7]. There are three type of disordering observed in the crystals of Ca-gallogermanate structural type. The first corresponds to disorder of structure appearing in one of the Wyckoff positions (there are two positions $2 d$ and $1 a$ ). This is typical for, e.g., $\mathrm{La}_{3} \mathrm{Ga}_{5} \mathrm{SiO}_{14}$ and $\mathrm{La}_{3} \mathrm{Ga}_{5,5} \mathrm{Ta}_{0.5} \mathrm{O}_{14}$ crystals [4]. On the other hand, there are two Wyckoff positions, $2 d$ and $3 f$, which are occupied by mixed $\mathrm{Ga}$ and $\mathrm{Ge}$ atoms in $\mathrm{Ca}_{3} \mathrm{Ga}_{2} \mathrm{Ge}_{4} \mathrm{O}_{14}[4]$.

According to study [7], the crystal lattice parameters of the langasite crystals are $a=8.175 \AA$ and $c=5.105 \AA$. In our calculations we have used the following electronic polarizability volumes: $\alpha_{\mathrm{La}}^{\prime}=1.886 \AA^{3}, \alpha_{\mathrm{Ga}}^{\prime}=0.375 \AA^{3}, \alpha_{\mathrm{Si}}^{\prime}=0.050 \AA^{3}$, and $\alpha_{\mathrm{O}}^{\prime}=1.740 \AA^{3}$. The sight occupation factor (S.O.F.) of $\mathrm{Ga}$ atom in the Wyckoff position $2 d$ varies from 0.4 to 0.6 , whereas the S.O.F. for $\mathrm{Si}$ atoms can be calculated as S.O.F.(Si) $=1-$ S.O.F.(Ga). Here the information on the crystalline structure reported in Ref. [7] (the S.O.F. for both of $\mathrm{Ga}$ and Si atoms being equal to 0.5) is used as the initial data. Finally, the polarizability volumes for the both $\mathrm{Ga}$ and $\mathrm{Si}$ atoms occupying the $2 d$ position have been determined as $\alpha_{\mathrm{GaSi}}^{\prime}=$ S.O.F.(Ga) $\alpha_{\mathrm{Ga}}^{\prime}+$ S.O.F.(Si) $\alpha_{\mathrm{Si}}^{\prime}$.

The main results of our calculations are displayed in Fig. 4. As seen from Fig. 4, the dependence of $\mathrm{OR}$ on the S.O.F. for $\mathrm{Ga}$ and $\mathrm{Si}$ in the $2 d$ Wickoff positions is linear. It is worthwhile that the OR can considerably (approximately by $0.8 \mathrm{deg} / \mathrm{mm}$ ) deviate from its 'nominal' value $\rho=3.0 \mathrm{deg} / \mathrm{mm}$, which is calculated while assuming the occupation $0.5 \mathrm{Ga}+0.5 \mathrm{Si}$ of the $2 d$ position. Furthermore, the OR increases with increasing S.O.F. for $\mathrm{Si}$ atoms or decreasing S.O.F. for Ga atoms. We are also to stress that $\alpha_{\mathrm{Ga}}^{\prime}>\alpha_{\mathrm{Si}}^{\prime}$ for the LGS crystals. 


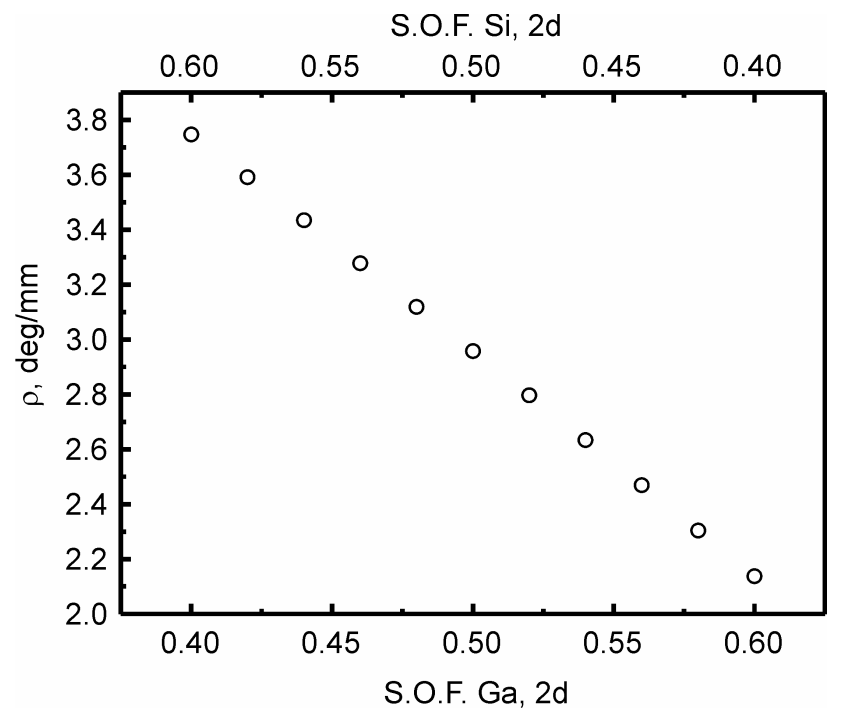

Fig. 4. OR as a function of S.O.F. for $\mathrm{Ga}$ and $\mathrm{Si}$ in the $2 d$ positions of LGS crystals. Calculations are performed for the light wavelength of $635 \mathrm{~nm}$.

In contrast to the above data for the optical activity, the refractive indices calculated according to the same procedures are found to remain almost invariable under the same changes in the S.O.F. for $\mathrm{Ga}$ and $\mathrm{Si}$ atoms: $n_{o}=1.900 \pm 0.002$ and $n_{e}=1.911 \pm 0.003$. Notice also that the refractive indices of LGS measured experimentally for the nearby laser light wavelength of 632.8 $\mathrm{nm}$ are close and equal to $n_{o}=1.8996$ and $n_{e}=1.9111[4,5]$.

\section{Conclusions}

We have experimentally found significant spatial variations in the OR parameter measured along the optic axis in LGS crystals. These changes cannot be explained by the linear birefringence induced with residual mechanical stresses typical for the crystals grown with the Czochralski technique. Meanwhile, our calculations have testified that the OR is very sensitive to structural inhomogeneities in the crystal. As a consequence, we assume that the effect of S.O.F. gives a dominant contribution to the spatial changes of OR along the $z$ axis.

\section{Acknowledgement}

The authors thank Dr. M. Dubovik (the Institute for Single Crystals, NAS of Ukraine) for kindly offering LGS crystals used in our measurements.

\section{References}

1. Hecht E, Optics ( ${ }^{\text {th }}$ Ed.). Harlow: Addison-Wesley, 2002.

2. Devarajan V and Glazer A, 1986. Theory and computation of optical rotatory power in inorganic crystals. Acta. Cryst. A. 42: 560-569.

3. Dubovik M F, 1992. Growing and properties of some oxide single crystals for multifunctional applications. Inorg. Mater. 28: 1999-2006.

4. Kaminski A A, Aminov LK, Ermolaev VL, Kornienko AA, Kravchenko VB, Malkin BZ, Mil BV, Perlin Yu E, Petrosyan AG, Pukhov KK, Sakun VP, Sarkisov SE, Sveshnikov EB, Skripko GA, Starostin NV, Shkaderevich AP, Physics and spectroscopy of laser crystals. Moscow: Nauka, 1986. 
5. Kaldybaev K A, Konstantinova A F and Perekalina Z B, Gyrotropy of uniaxial absorbing crystals. Moscow: ISPIN, 2000.

6. Fu X H, Che Y and Li Y L, 2011. Diode-pumped Nd: LGS intracavity-doublet green laser at 532 nm. Laser Phys. 21: 995-997.

7. Maksimov A A, Molchanov V N, Mill B V, Belokoneva E L, Rabadanov M Kh, Pugacheva A A, Pisarvskii $\mathrm{Yu} \mathrm{V}$ and Simonov V I, 2005. Absolute structure of $\mathrm{La}_{3} \mathrm{Ga}_{5} \mathrm{SiO}_{14}$ langasite crystals. Crystallogr. Rep. 50: 751-758.

8. Veremeichik T F, 2011. Optical activity and crystalline structure of crystals of the langasite family. Crystallogr. Rep. 56: 1060-1065.

9. Kitaura M, Mochizuki K, Inabe Y, Itoh M, Nakagava H and Oishi S, 2004. Fundamental optical properties and electronic structure of langasite $\mathrm{La}_{3} \mathrm{Ga}_{5} \mathrm{SiO}_{14}$ crystals. Phys. Rev. B. 69: 115120-1-115120-6.

10. Geras'kin V V, Klyukhina Yu V, Buzanov O A and Petrakov V S, 2007. Influence of optical inhomogeneity of langasite crystals on the value of measured electro-optic coefficient. Crystallogr. Rep. 52: 870-879.

11. Dubovskii A B, Tyunina E A, Domoroshchina E N, Kuz'micheva G M and Rybakov V B, 2008. Composition effect on the elastic properties of langasite. Inorg. Mater. 44: 520-526.

12. Jung, Water G, Op Amp applications handbook. Amsterdam: Elsevier, 2005.

13. Azzam R M A and Bashara N M, Ellipsometry and polarized light. Amsterdam: Elsevier, 2003.

14. Vlokh O G, Spatial dispersion phenomena in parametric crystal optics. Lviv: Vyshcha Shkola, 1984.

15. Konstantinova A F, Evdishchenko A E and Imangazieva K B, 2006. Manifestation of optical activity in crystals of different symmetry classes. Crystallogr. Rep. 51: 998-1008.

16. Born M and Huang K, Dynamical theory of crystal lattices. Oxford: Oxford University Press, 1998.

17. Shopa Ya and Ftomyn N, Calculation of optical rotation and refractive indices in the langasite family crystals. Proc. Internat. Conf. on Oxide Materials for Electron. Eng. (OMEE-2012). Nat. Univ. "Lviv Polytechnics" (2012) 137-138.

Shopa Ya., Ftomyn N. and Sokoliuk I. 2014. Crystal structure and optical activity of $\mathrm{La}_{3} \mathrm{Ga}_{5} \mathrm{SiO}_{14}$ crystals. Ukr.J.Phys.Opt. 15: 155 - 161.

Анотація. За допомогою лазерного поляриметра (635 нм) побудовано мапу просторового розподілу питомого оптичного повертання (ОП) для зразка Z-зрізу кристалів лангаситу $\mathrm{La}_{3} \mathrm{Ga}_{5} \mathrm{SiO}_{14}$ iз поперечними розмірами $3 \times 17$ мм $^{2} i$ товщиною 0,945 мм. Ураховано можливий вплив лінійного двозаломлення на вимірюване ОП. На основі теорії поляризованості для оптичної активності розраховано питоме ОП як функцію параметрів розупорядкування структури досліджуваного матеріалу. Показано, шэо структурне розупорядкування переважає в просторових змінах оптичної активності вздовж оптичної осі. 